JHU-TIPAC-96007

hep-ph/9603389

\title{
A New Interpretation of the Observed Heavy Baryons
}

\author{
Adam F. Falk \\ Department of Physics and Astronomy, The Johns Hopkins University \\ 3400 North Charles Street, Baltimore, Maryland 21218
}

(March 1996)

\begin{abstract}
I suggest that the conventional assignment of quantum numbers to the observed charm and bottom baryons is not correct, as these assignments imply large violation of the heavy spin-flavor and light $S U(3)$ symmetries. I propose an alternative interpretation of the observed states, in which the symmetries are preserved. If these novel assignments are right, there is a new state with mass approximately $2380 \mathrm{MeV}$ which decays to $\Lambda_{c}+\gamma$, and another with mass approximately $5760 \mathrm{MeV}$ which decays to $\Lambda_{b}+\gamma$. Although such states have not been seen, neither are they excluded by current analyses.
\end{abstract}

Typeset using REVTEX 
The past few years have seen the discovery of many new hadrons containing a single charm or bottom quark. Such states fall into representations of heavy quark spin-flavor $S U(4)$ and light flavor $S U(3)$ symmetries, up to heavy quark corrections of order $\Lambda_{\mathrm{QCD}} / 2 m_{Q}$ and $S U(3)$ corrections of order $m_{q} / \Lambda_{\chi}$. Enough have now been discovered to make possible detailed tests of the relations implied by the symmetries. In the heavy meson sector, these predictions are known to work well for the ground states and the lowest $P$-wave excitations [1]. Not only the spectroscopy, but the widths and even the decay angular distributions are consistent with a simultaneous heavy quark and chiral $S U(3)$ expansion. Hence one is tempted to hope that the symmetry predictions for heavy baryons are also well satisfied. However, in contrast to the mesons, for the baryons there are certain symmetry relations which appear to be badly violated, although others appear to work well.

While it is possible that the symmetry breaking corrections are just larger than expected, such an explanation would offer no insight into why some relations behave better than others. In this letter, I will propose that the problem is instead that the conventional assignment of quantum numbers to the observed charm and bottom baryons is not correct. I will show how one can satisfy all the symmetry relations at the expected level by assigning new quantum numbers to the known resonances. An exciting consequence is the existence of additional light excitations which only decay radiatively. Such states are not presently ruled out, and this prediction presents a well defined and conclusive test of the proposal.

I begin with a review of baryon spectroscopy in the heavy quark limit, $m_{c}, m_{b} \rightarrow \infty$. In this limit, heavy quark pair production and chromomagnetic interactions are suppressed, so the angular momentum and flavor quantum numbers of the light degrees of freedom become good quantum numbers. I will refer to these light degrees of freedom as a "diquark"; in doing so, I assume nothing about their properties other than that they carry certain spin and flavor quantum numbers. For simplicity, I will also restrict myself for the moment to heavy charm baryons, since the enumeration of states for bottom baryons is precisely analogous.

In the quark model, the lightest diquark has isospin $I=0$, total spin $s_{\ell}=0$ and orbital angular momentum $L_{\ell}=0$. With diquark spin-parity $J_{\ell}^{P}=0^{+}$, this leads to the heavy baryon $\Lambda_{c}$, with total $J^{P}=\frac{1}{2}^{+}$. The strange analogue of the $\Lambda_{c}$ is the $\Xi_{c}$, with $I=\frac{1}{2}$. Because of Fermi statistics, there is no doubly strange state with $s_{\ell}=0$. There is a nearby excitation of the $\Lambda_{c}$, in which the diquark is in the same orbital state, but with $I=s_{\ell}=1$. This leads to a doublet of heavy baryons consisting of the $\Sigma_{c}$, with $J^{P}=\frac{1}{2}^{+}$, and the $\Sigma_{c}^{*}$, with $J^{P}=\frac{3}{2}^{+}$. As with all heavy doublets, the chromomagnetic hyperfine splitting between these states is of order $\Lambda_{\mathrm{QCD}}^{2} / m_{c}$. The strange analogues of the $\Sigma_{c}$ and $\Sigma_{c}^{*}$ are respectively the $\Xi_{c}^{\prime}$ and $\Xi_{c}^{*}$, and there are also the doubly strange states $\Omega_{c}$ and $\Omega_{c}^{*}$.

The diquark may be excited further by adding a unit of orbital angular momentum, $L_{\ell}=1$. More precisely, this is true in the constituent quark model, which guides our intuition that resonances with these quantum numbers might be close by. When $I=s_{\ell}=0$, the excited diquark has total spin-parity $J_{\ell}^{P}=1^{-}$, and the heavy baryon states are the $\Lambda_{c}^{*}\left(\frac{1}{2}\right)$ and the $\Lambda_{c}^{*}\left(\frac{3}{2}\right)$. When $I=s_{\ell}=1$, one finds diquarks with $J_{\ell}^{P}=0^{-}, 1^{-}$and $2^{-}$, leading to the odd parity heavy baryons $\Sigma_{c 0}^{*}, \Sigma_{c 1}^{*}\left(\frac{1}{2}, \frac{3}{2}\right)$ and $\Sigma_{c 2}^{*}\left(\frac{3}{2}, \frac{5}{2}\right)$. There are also excited $\Xi_{c}$ and $\Omega_{c}$ baryons. The spectroscopy of the charm baryons is summarized in Table \&, along with the allowed decays of the states. Two channels are listed where there is the possibility that either is kinematically dominant.

The masses of these states satisfy a number of heavy quark and $S U(3)$ symmetry rela- 


\begin{tabular}{llllllll}
\hline \hline Name & $J^{P}$ & $s_{\ell}$ & $L_{\ell}$ & $J_{\ell}^{P}$ & $I$ & $S$ & Decay \\
\hline \hline$\Lambda_{c}$ & $\frac{1}{2}^{+}$ & 0 & 0 & $0^{+}$ & 0 & 0 & weak \\
$\Sigma_{c}$ & $\frac{1}{2}^{+}$ & 1 & 0 & $1^{+}$ & 1 & 0 & $\Lambda_{c} \gamma, \Lambda_{c} \pi$ \\
$\Sigma_{c}^{*}$ & $\frac{3}{2}^{+}$ & 1 & 0 & $1^{+}$ & 1 & 0 & $\Lambda_{c} \pi$ \\
$\Xi_{c}$ & $\frac{1}{2}^{+}$ & 0 & 0 & $0^{+}$ & $\frac{1}{2}$ & -1 & weak \\
$\Xi_{c}^{\prime}$ & $\frac{1}{2}^{+}$ & 1 & 0 & $1^{+}$ & $\frac{1}{2}$ & -1 & $\Xi_{c} \gamma, \Xi_{c} \pi$ \\
$\Xi_{c}^{*}$ & $\frac{3}{2}^{+}$ & 1 & 0 & $1^{+}$ & $\frac{1}{2}$ & -1 & $\Xi_{c} \pi$ \\
$\Omega_{c}$ & $\frac{1}{2}^{+}$ & 1 & 0 & $1^{+}$ & 0 & -2 & weak \\
$\Omega_{c}^{*}$ & $\frac{3}{2}^{+}$ & 1 & 0 & $1^{+}$ & 0 & -2 & $\Omega_{c} \gamma$ \\
$\Lambda_{c}^{*}\left(\frac{1}{2}\right)$ & $\frac{1}{2}^{-}$ & 0 & 1 & $1^{-}$ & 0 & 0 & $\Sigma_{c} \pi, \Lambda_{c} \pi \pi$ \\
$\Lambda_{c}^{*}\left(\frac{3}{2}\right)$ & $\frac{3}{2}^{-}$ & 0 & 1 & $1^{-}$ & 0 & 0 & $\Sigma_{c}^{*} \pi, \Lambda_{c} \pi \pi$ \\
$\Sigma_{c 0}^{*}$ & $\frac{1}{2}^{-}$ & 1 & 1 & $0^{-}$ & 1 & 0 & $\Lambda_{c} \pi$ \\
$\Sigma_{c 1}^{*}\left(\frac{1}{2}, \frac{3}{2}\right)$ & $\frac{1}{2}^{-}, \frac{3}{2}^{-}$ & 1 & 1 & $1^{-}$ & 1 & 0 & $\Lambda_{c} \pi$ \\
$\Sigma_{c 2}^{*}\left(\frac{3}{2}, \frac{5}{2}\right)$ & $\frac{3}{2}^{-}, \frac{5}{2}^{-}$ & 1 & 1 & $2^{-}$ & 1 & 0 & $\Lambda_{c} \pi$ \\
\hline \hline
\end{tabular}

TABLE I. Charm baryon states in the heavy quark limit. Here $s_{\ell}, L_{\ell}$ and $J_{\ell}^{P}$ refer respectively to the spin, orbital angular momentum, and total spin-parity of the light diquark, while $I$ is isospin and $S$ strangeness. The given decay channel is the one which is expected to be dominant, if kinematically allowed. The enumeration of the bottom baryon states is analogous.

tions. There are three independent constraints which relate the bottom and charm systems,

$$
\begin{aligned}
& \Lambda_{b}-\Lambda_{c}=\bar{B}-\bar{D}=3340 \mathrm{MeV}, \\
& \bar{\Sigma}_{b}-\Lambda_{b}=\bar{\Sigma}_{c}-\Lambda_{c}, \\
& \frac{\Sigma_{b}^{*}-\Sigma_{b}}{\Sigma_{c}^{*}-\Sigma_{c}}=\frac{B^{*}-B}{D^{*}-D}=0.33,
\end{aligned}
$$

where in (12a) and (1.C) I have inserted the isospin averaged heavy meson masses [2]. Here the states stand for their masses, and a bar over a state denotes the spin average over the heavy multiplet of which it is a part. This spin average, which cancels the hyperfine interaction between the heavy quark and the collective light degrees of freedom, takes the form $\left(D+3 D^{*}\right) / 4$ for the ground state heavy mesons and $\left(\Sigma_{c}+2 \Sigma_{c}^{*}\right) / 3$, etc., for the spin$\left(\frac{1}{2}, \frac{3}{2}\right)$ heavy baryon doublets. The hyperfine relation (1T) is more commonly written in terms of the ratio $m_{c} / m_{b}$, to which each side is equal, but I prefer a form in which the quark masses are not introduced explicitly. The corrections to (11a) and (11b) are expected to be of order $\Lambda_{\mathrm{QCD}}^{2}\left(1 / 2 m_{c}-1 / 2 m_{b}\right) \sim 50 \mathrm{MeV}$. The corrections to (1) could be at the level of $25 \%$.

The light flavor $S U(3)$ relations are trivial in the exact symmetry limit, where, for example, $\Sigma_{c}=\Xi_{c}^{\prime}=\Omega_{c}$. In this form, they are also badly violated. If one includes the corrections linear in $m_{s}$, one finds four independent "equal spacing rules" for states within the charm (or bottom) system [3],

$$
\begin{aligned}
& \Omega_{c}-\Xi_{c}^{\prime}=\Xi_{c}^{\prime}-\Sigma_{c}, \\
& \Omega_{c}^{*}-\Xi_{c}^{*}=\Xi_{c}^{*}-\Sigma_{c}^{*}, \\
& \Sigma_{c}^{*}-\Sigma_{c}=\Xi_{c}^{*}-\Xi_{c}^{\prime}=\Omega_{c}^{*}-\Omega_{c}, \\
& \Sigma_{c}-\Lambda_{c}=\Xi_{c}^{*}-\Xi_{c} .
\end{aligned}
$$




\begin{tabular}{|c|c|c|c|c|c|}
\hline State & Mass $(\mathrm{MeV})$ & Ref. & Decay Channel & Conventional & Proposed \\
\hline \multirow[t]{2}{*}{$\overline{\Lambda_{c}}$} & $2285 \pm 1$ & 2 & weak & $\Lambda_{c}$ & $\overline{\Lambda_{c}}$ \\
\hline & $(2380)$ & & $\Lambda_{c}+\gamma$ & absent & $\Sigma_{c}$ \\
\hline$\Sigma_{c 1}$ & $2453 \pm 1$ & 2 & $\Lambda_{c}+\pi$ & $\Sigma_{c}$ & $\Sigma_{c}^{*}$ \\
\hline$\Sigma_{c 2}$ & $2530 \pm 5 \pm 5$ & 何 & $\Lambda_{c}+\pi$ & $\Sigma_{c}^{*}$ & $\Sigma_{c 0}^{*}(?)$ \\
\hline$\Xi_{c}$ & $2468 \pm 2$ & 2] & weak & $\Xi_{c}$ & $\Xi_{c}$ \\
\hline$\Xi_{c 1}$ & $2563 \pm 15(?)$ & {$[5]^{\mathrm{a}}$} & $\Xi_{c}+\gamma$ & $\Xi_{c}^{\prime}$ & $\Xi_{c}^{\prime}$ \\
\hline$\Xi_{c 2}$ & $2644 \pm 2$ & 6 & $\Xi_{c}+\pi$ & $\Xi_{c}^{*}$ & $\Xi_{c}^{*}$ \\
\hline$\Omega_{c}$ & $2700 \pm 3$ & [7] & weak & $\Omega_{c}$ & $\Omega_{c}$ \\
\hline$\Lambda_{c 1}^{*}$ & $2593 \pm 1$ & {$[2,8]$} & $\Sigma_{c 1}+\pi \rightarrow \Lambda_{c}+2 \pi$ & $\Lambda_{c}^{*}\left(\frac{1}{2}\right)$ & $\Lambda_{c}^{*}\left(\frac{3}{2}\right)$ \\
\hline$\Lambda_{c 2}^{*}$ & $2627 \pm 1$ & 8 & $\Lambda_{c}+\pi+\pi$ & $\Lambda_{c}^{*}\left(\frac{3}{2}\right)$ & $\Lambda_{c}^{*}\left(\frac{1}{2}\right)$ \\
\hline \multirow[t]{2}{*}{$\overline{\Lambda_{b}}$} & $5623 \pm 5 \pm 4$ & [2,, 9$]$ & weak & $\Lambda_{b}$ & $\Lambda_{b}$ \\
\hline & $(5760)$ & & $\Lambda_{b}+\gamma$ & absent & $\Sigma_{b}$ \\
\hline$\Sigma_{b 1}$ & $5796 \pm 3 \pm 5$ & 10 & $\Lambda_{b}+\pi$ & $\Sigma_{b}$ & $\Sigma_{b}^{*}$ \\
\hline$\Sigma_{b 2}$ & $5852 \pm 3 \pm 5$ & 10] & $\Lambda_{b}+\pi$ & $\Sigma_{b}^{*}$ & $\Sigma_{b 0}^{*}(?)$ \\
\hline
\end{tabular}

TABLE II. The observed heavy baryon states, with their conventional and proposed identities. Isospin multiplets have been averaged over. Experimental errors ( \pm stat. \pm sys.) are included where significant; where they are small, statistical and systematic errors have, for simplicity, been added in quadrature. The approximate masses of the proposed new states are given in parentheses.

aThe mass of the $\Xi_{c 1}$ is estimated from the plots presented by WA89. Only one of the two isospin states has been observed.

Here I neglect isospin violation and electromagnetic effects. The chiral corrections to the relations (2a) $-(2 \mathrm{~d})$ are expected to be small [3]. The relation (2d) is not on the same footing as the others, since it relates states in two different $S U(3)$ multiplets. It is actually a combined $S U(3)$ and heavy quark symmetry relation. The leading corrections to it are, in principle, of order $m_{s}$, and cannot be calculated. However, one's intuition from the quark model is that this relation should be reasonably well satisfied, and indeed the counterparts in the charmed meson sector, such as $D_{s 1}-D_{s}=D_{1}-D$, work to within $10 \mathrm{MeV}$. In fact all of the heavy quark and $S U(3)$ relations for the charm and bottom mesons work beautifully [1].

So far, a dozen charm and bottom baryon states have been discovered. I list them, along with their masses and observed decays, in Table 【. However, the names conventionally given to the strongly decaying states imply certain assumptions about their quantum numbers and properties. Since it is precisely these assumptions which I want to challenge, I instead identify the observed resonances by the modified names listed in the first column of Table [I]. For simplicity, I have averaged over isospin multiplets, since isospin breaking is small and not at issue here.

The conventional identities of the observed heavy baryons are given in the fourth column of Table 【I. How well do the predictions of heavy quark and $S U(3)$ symmetry fare? The heavy quark constraints ([a) and ([1]) are both satisfied to within $10 \mathrm{MeV}$. However, the hyperfine relation $(1 \mathrm{~d})$ is in serious trouble. One finds $\left(\Sigma_{b}^{*}-\Sigma_{b}\right) /\left(\Sigma_{c}^{*}-\Sigma_{c}\right) \approx 0.73 \pm 0.13$, too large by a factor of two! To be conservative, I have ignored the correlation between the errors on the $\Sigma_{b}$ and the $\Sigma_{b}^{*}$, hence overestimating the total uncertainty. It is clear that to 
take these data seriously is to identify a crisis for the application of heavy quark symmetry to the charm and bottom baryons.

Neither is the situation perfect for the $S U(3)$ relations. The first equal spacing rule (2a), with the well measured masses of the $\Sigma_{c}$ and the $\Omega_{c}$, yields the prediction $\Xi_{c}^{\prime}=2577 \mathrm{MeV}$, somewhat large but probably within the experimental error. The second rule (2b) cannot be tested, as the $\Omega_{c}^{*}$ state has not yet been found. Inserting the measured $\Sigma_{c}, \Sigma_{c}^{*}$ and $\Xi_{c}^{*}$ masses, the third rule (2G) may be rearranged to yield the prediction $\Xi_{c}^{\prime}=2567 \mathrm{MeV}$, reasonably consistent both with (2a) and with experiment. However, the final $S U(3)$ relation (2d) fails by approximately $80 \mathrm{MeV}$, an order of magnitude worse than for the charmed mesons! Such an enormous discrepancy is quite surprising and disappointing.

What are we to make of this situation, in which one heavy quark and one $S U(3)$ relation fail so badly? Given that there is no reason to doubt the quoted experimental errors, perhaps we must simply accept that there are large corrections, that somehow these important symmetries are inapplicable to heavy baryons. However, with their striking success in the heavy meson sector, especially for spectroscopy, it is tempting to look for a new point of view from which the symmetry predictions are better behaved.

In this light, I propose to reinterpret the experimental data under the constraint that the heavy quark and $S U(3)$ symmetries be imposed explicitly. Then if we identify, once again, the observed $\Xi_{c 1}$ with the $\Xi_{c}^{\prime}$ state, the $S U(3)$ relations (2) lead to the novel mass prediction $\Sigma_{c} \approx 2380 \mathrm{MeV}$ ! If so, the $\Sigma_{c}$ cannot be identified with the observed $\Sigma_{c 1}$; in fact, it can be identified with no resonance yet to have been reported. However, since at this mass the $\Sigma_{c}$ can decay only radiatively, $\Sigma_{c} \rightarrow \Lambda_{c}+\gamma$, it is quite possible that it exists but so far has been overlooked.

The observed $\Sigma_{c 1}$ is now identified as the $\Sigma_{c}^{*}$. In the bottom baryons, there is a similar reassignment: the $\Sigma_{b}$ is now assumed to be below $\Lambda_{b}+\pi$ threshold and to decay radiatively, while the $\Sigma_{b 1}$ is identified as the $\Sigma_{b}^{*}$. As for the observed $\Sigma_{c 2}$ and $\Sigma_{b 2}$, they are possibly $I=1$, $L_{\ell}=1$ excitations, such as the $\Sigma_{c(0,1,2)}^{*}$. While one might naively estimate that the masses of these states should be larger than those of the $\Lambda_{c}^{*}\left(\frac{1}{2}\right)$ and $\Lambda_{c}^{*}\left(\frac{3}{2}\right)$, a substantial spin-orbit coupling could lower the mass of the state $\Sigma_{c 0}^{*}$ by of order $200 \mathrm{MeV}$. Hence I tentatively identify the observed $\Sigma_{c 2}$ and $\Sigma_{b 2}$ respectively as the $\Sigma_{c 0}^{*}$ and $\Sigma_{b 0}^{*}$.

The poorly behaved symmetry relations improve dramatically in this scenario. For example, let us take the masses of the new states to be $\Sigma_{c}=2380 \mathrm{MeV}$ and $\Sigma_{b}=5760 \mathrm{MeV}$. Then the hyperfine splitting ratio (1c) improves to $\left(\Sigma_{b}^{*}-\Sigma_{b}\right) /\left(\Sigma_{c}^{*}-\Sigma_{c}\right)=0.49$, and the $S U(3)$ relation $(2 \mathrm{~d})$ between the $s_{\ell}=0$ and $s_{\ell}=1$ states is satisfied to within $5 \mathrm{MeV}$. The heavy quark relation (1a) is unaffected, while the constraint (1b) for the $\bar{\Sigma}_{Q}$ excitation energy is satisfied to within $20 \mathrm{MeV}$, which is quite reasonable. Only the $S U(3)$ equal spacing rules (2a) and (2c) suffer mildly from the change. Taken, as before, as a prediction for the mass of the $\Xi_{c}^{\prime}$, the former relation now fails by $23 \mathrm{MeV}$. The latter now fails by $8 \mathrm{MeV}$, but the discrepancies are in opposite directions, and the two relations cannot be satisfied simultaneously by shifting the mass of the $\Xi_{c}^{\prime}$. With these new assignments, intrinsic $S U(3)$ violating corrections of the order of $15 \mathrm{MeV}$ seem to be unavoidable.

With respect to the symmetry predictions as a whole, the new scenario is an enormous improvement over the old. The heavy quark and $S U(3)$ flavor symmetries have been resurrected. We can improve the agreement further if we allow the measured masses to vary within their reported $1 \sigma$ errors. One set of allowed masses is $\Sigma_{c}=2375 \mathrm{MeV}, \Sigma_{c}^{*}=2453 \mathrm{MeV}$, 
$\Xi_{c}^{\prime}=2553 \mathrm{MeV}, \Xi_{c}^{*}=2644 \mathrm{MeV}, \Sigma_{b}=5760 \mathrm{MeV}$, and $\Sigma_{b}^{*}=5790 \mathrm{MeV}$. For this choice, the $S U(3)$ relations (2a) and (2c) (taken as predictions for the $\Xi_{c}^{\prime}$ mass) and (2d) are satisfied to within $15 \mathrm{MeV}, 13 \mathrm{MeV}$ and $4 \mathrm{MeV}$, respectively. The hyperfine ratio (10) is $\left(\Sigma_{b}^{*}-\Sigma_{b}\right) /\left(\Sigma_{c}^{*}-\Sigma_{c}\right)=0.38$, and $\bar{\Sigma}_{b}-\Lambda_{b}$ is equal to $\bar{\Sigma}_{c}-\Lambda_{c}$ to within $15 \mathrm{MeV}$. This is better agreement with the symmetries than we even have a right to expect.

As appealing a scenario as this is, certain problems do remain. First, while the radiatively decaying states $\Sigma_{c}$ and $\Sigma_{b}$ have not been ruled out, neither have they yet been identified. In the end, their discovery or the absence thereof will be the defining test of this proposal. Second, the excited baryon $\Lambda_{c 1}^{*}$ is seen to decay via the two step process $\Lambda_{c 1}^{*} \rightarrow \Sigma_{c 1}+\pi \rightarrow$ $\Lambda_{c}+2 \pi$, while the two pion decay of the $\Lambda_{c 2}^{*}$ is nonresonant [6]. If the observed states $\Lambda_{c 1}^{*}$ and $\Lambda_{c 2}^{*}$ are identified with the heavy doublet $\Lambda_{c}^{*}\left(\frac{1}{2}\right)$ and $\Lambda_{c}^{*}\left(\frac{3}{2}\right)$, then the first stage in the decay of the $\Lambda_{c 1}^{*}$ is dominated by $S$-wave pion emission [11]. If so, the spin of the $\Lambda_{c 1}^{*}$ is the same as that of the $\Sigma_{c 2}$, namely $J=\frac{3}{2}$. Hence the excited $I=0$ doublet must be inverted, with $\Lambda_{c}^{*}\left(\frac{3}{2}\right)<\Lambda_{c}^{*}\left(\frac{1}{2}\right)$. Perhaps this situation is somewhat unnatural, perhaps not.

However, the least satisfactory feature of this scenario is the identification of the $\Sigma_{b 2}$ as the $\Sigma_{b 0}^{*}$ state, with $s_{\ell}=L_{\ell}=1$ and $J_{\ell}^{P}=0^{-}$. The DELPHI analysis [10 of the masses, production and decay properties of the $\Sigma_{b 1}$ and $\Sigma_{b 2}$ explains in an elegant and nontrivial manner the surprisingly low observed polarization of $\Lambda_{b}$ 's produced at the $Z^{0}$. [12, 13]. The analysis was predicated, of course, on the conventional assignment of quantum numbers; now this nice explanation of $\Lambda_{b}$ depolarization is lost. Worse, while the $S$-wave decay $\Sigma_{b 0}^{*} \rightarrow \Lambda_{b}+\pi$ must be isotropic, there appears to be a large anisotropy in the direction of the pion in $\Sigma_{b 2} \rightarrow \Lambda_{b}+\pi$ [10]. The reported deviation from an isotropic distribution is about $2.5 \sigma$. If this result is confirmed, the observed $\Sigma_{b 2}$ state must be something else, such as a radial excitation of the $\Sigma_{b}^{*}$.

Finally, it is worth noting that nonrelativistic consituent quark models (see, for example, the many papers cited in Ref. [3]) typically do not favor such a light $\Sigma_{c}$ and $\Sigma_{c}^{*}$ as I have suggested here. In fact, such models cannot be reconciled simultaneously with the heavy quark limit and with the reported masses of the $\Sigma_{b}$ and $\Sigma_{b}^{*}$. Hence, the predictions of this letter follow experiment in pointing to physics beyond the constituent quark model. While the historical usefulness of this model for hadron spectroscopy may well lead one to be suspicious of the $\Sigma_{b}$ and $\Sigma_{b}^{*}$ data, such speculation is beyond the scope of this discussion. I have taken the masses and errors of all states as they have been reported to date; as they evolve in the future, so, of course, will the theoretical analysis.

While such issues are important, the smoking gun here is the prediction of new heavy baryon excitations of approximately $100 \mathrm{MeV}$, decaying radiatively to $\Lambda_{c}$ and $\Lambda_{b}$. If confirmed, this will be the most unexpected and striking prediction yet to be obtained from heavy quark symmetry. If not, and if the reported data are correct, we will have to accept the failure of heavy spin-flavor and light $S U(3)$ symmetry to describe the charm and bottom baryon states.

It is a pleasure to thank Jon Bagger, Mike Booth, Bob Fletcher, Mike Luke and Tom Mehen for helpful conversations, and John Yelton and Don Fujino for invaluable correspondence concerning the CLEO experiment. This work was supported by the National Science Foundation under Grant No. PHY-9404057 and National Young Investigator Award No. PHY-9457916, by the Department of Energy under Outstanding Junior Investigator Award No. DE-FG02-94ER40869, and by the Alfred P. Sloan Foundation. 


\section{REFERENCES}

[1] For a review of heavy mesons, see A. F. Falk and T. Mehen, Phys. Rev. D53, 231 (1996).

[2] Particle Data Group, Review of Particle Properties, Phys. Rev. D50, 1173 (1994).

[3] M. J. Savage, Phys. Lett. B359, 189 (1995).

[4] SKAT Collaboration (V. A. Ammosov et al.), Pis'ma Zh. Eksper. Teor. Fiz. 58, 241 (1993).

[5] WA89 Collaboration, "Charmed Baryon Production in the CERN Hyperon Beam", presented by E. Chudakov at Heavy Quarks '94, Virginia, October, 1994.

[6] CLEO Collaboration (P. Avery et al.), CLNS-95-1352 (1995); CLNS-96-1394 (1996).

[7] E687 Collaboration (P. Frabetti et al.), Phys. Lett. B338, 106 (1994).

[8] CLEO Collaboration (K. W. Edwards et al.), Phys. Rev. Lett. 74, 3331 (1995).

[9] CDF Collaboration, CDF note 3352, available at http://www-cdf.fnal.gov/physics/ new/bottom/cdf3352.html.

[10] DELPHI Collaboration (M. Feindt et al.), CERN-PPE/95-139.

[11] P. Cho, Phys. Rev. D50, 3295 (1994).

[12] ALEPH Collaboration (D. Buskulic et al.), Phys. Lett. 365, 437 (1996).

[13] A. F. Falk and M. E. Peskin, Phys. Rev. D49, 3320 (1994). 